\title{
Estrategias logísticas orientadas al mejoramiento de la gestión de almacenamiento en empresas
}

\author{
Logistics strategies oriented to the improvement of the storage \\ management of a company
}

\author{
Audris Amaro \\ audris_30@hotmail.com \\ Código ORCID: 0000-0002-7016-5033 \\ Universidad de Carabobo - Venezuela
}

\section{Tábata Cisneros}

cisnerostabata@gmail.com

Código ORCID: 0000-0001-5900-4266

Universidad de Carabobo - Venezuela

\author{
Dylan Pérez \\ perezdylan@hotmail.com \\ Código ORCID: 0000-0002-8527-8241 \\ Universidad de Carabobo - Venezuela
}

Artículo recibido abril 2019 / Arbitrado en mayo 2019 / Publicado en julio 2019

\section{RESUMEN}

El estudio presentado tiene como objetivo general diseñar estrategias logísticas orientadas al mejoramiento de la gestión de almacenamiento de la empresa Molinari \& Cacciaguerra Sucrs, C. A, en la que se manifiestan constantes problemas con las condiciones de almacenaje, que genera devoluciones por daños en el estado de los productos, lo que se traduce en el incumplimiento de los compromisos adquiridos con sus clientes y la poca satisfacción de los clientes. Metodológicamente, fue concebida como una investigación descriptiva de diseño de campo y con modalidad de proyecto factible, en la que se empleó como técnicas de recolección de datos la guía de observación y la encuesta. De acuerdo a los resultados obtenidos, se propuso un plan de acciones estratégicas basadas en la organización del área de almacén de la empresa de acuerdo a los criterios y niveles de actividad previstos y sus expectativas de crecimiento.

Palabras clave: Estrategias logísticas; gestión de almacenamiento; manejo de inventario; indicadores de gestión

\begin{abstract}
The study presented has the general objective of designing logistics strategies aimed at improving the storage management of the company Molinari \& Cacciaguerra Sucrs, C. A, in which there are constant problems with storage conditions, which generates returns for damage to the status of the products, which translates into noncompliance with the commitments acquired with its customers and low customer satisfaction. Methodologically, it was conceived as a descriptive research of field design and with feasible project modality, in which the observation guide and the survey were used as data collection techniques. According to the results obtained, a strategic action plan was proposed based on the organization of the company's warehouse area according to the criteria and levels of activity foreseen and its growth expectations.
\end{abstract}

Keywords: Logistic strategies; storage management; good warehouse practices; management indicators 


\section{INTRODUCCIÓN}

La complejidad del entorno donde se desarrollan las organizaciones en la actualidad, requiere de una revisión permanente de sus actividades, debido a su influencia relevante en la decisión estratégica de la organización; una de las alternativas que tienen los directivos para conseguir este propósito es la aplicación de un sistema de dirección con enfoque estratégico. El problema se presenta, de acuerdo con Müller (2011:32), "debido a que la brecha existente entre la capacidad de las organizaciones para formular y ejecutar estrategias y su capacidad de aprender de dicho proceso ha crecido y precisa ser reducida con urgencia". Para ello, el autor comenta que se requiere desarrollar un aprendizaje estratégico desde el interior de la organización, que permita modificar decisiones ya adoptadas, sin que se generen conflictos ni falta de alineación con la visión trazada.

En este sentido, dentro del conjunto de estrategias que se pueden desarrollar en una organización, las orientadas hacia la eficiencia operacional en sus procesos logísticos están dirigidas, de acuerdo con Serna Gómez (2009:16), a "realizar un conjunto de actividades competitivas y capaces de reaccionar autónomamente a los cambios tecnológicos y las tendencias del entorno", mediante el control constante de la capacidad de cada proceso, la mejora continua, inductores de medición de desempeño, flexibilidad estructural y la orientación de las actividades hacia la plena satisfacción del cliente y de sus necesidades, así como de proveer normas de control a la organización. Es uno de los mecanismos más efectivos para que la organización alcance unos altos niveles de eficiencia.

Por tal motivo, la calidad del servicio logístico se convierte en un elemento estratégico que confiere una ventaja diferenciadora y perdurable en el tiempo a aquellas entidades que tratan de alcanzarla., Steimberg (2008:38) indica que "la gestión de calidad que debe generarse en el proceso de intercambio que la gestión logística supone, y más concretamente la calidad del servicio, se está convirtiendo en requisito imprescindible para competir en las organizaciones de todo el mundo", ya que las implicaciones que tiene la logística sobre resultados de las organizaciones, tanto en corto como en largo plazo, son muy positivas para las entidades envueltas en este tipo de procesos.

Dentro de este contexto, entre los elementos que forman la estructura del sistema logístico, en las empresas industriales o comerciales, la gestión de almacén es una de las funciones que participa en el control de inventario, el abastecimiento y la distribución física, constituyendo una de las actividades más importantes para el funcionamiento de la empresa; sin embargo, muchas veces fue olvidada por considerársele como el depósito donde se guardaban los materiales que requerían las áreas de producción o ventas.

Su dependencia de los departamentos mencionados, se basaba en la necesidad de contar con los materiales y por eso se destinaba a trabajar en el personal de confianza de los dirigentes. Esa situación ha cambiado y los estudios de la logística actuales le dan una especial importancia dentro de la cadena de suministro de una organización. Por ello, el almacén es definido por Chase, Jacobs 
y Aquilano (2009:115), como "una unidad de servicio en la estructura orgánica y funcional de una empresa comercial o industrial, con objetivos bien definidos de resguardo, custodia, control y abastecimiento de materiales y productos". Debido a ello, es indispensable conocer y aplicar las herramientas y técnicas más avanzadas que permitan facilitar las tareas administrativas y humanas en los diferentes procesos de la administración de almacenes e inventarios.

Es significativo indicar que, en Venezuela, al igual que en el mundo entero, el auge empresarial es cada día más significativo, porque las organizaciones frecuentemente manejan una amplia variedad de procesos administrativos y operacionales, las cuales una vez que son llevadas a cabo resultan totalmente eficientes y efectivas para el desarrollo de las empresas. En este sentido, las empresas nacionales tratan de establecer una adecuada estructura, ya que es de gran importancia, para la organización final de divisiones y departamentos funcionales, de igual manera, la asignación de responsabilidades y políticas de delegación de autoridad, y características laborales $u$ organizativas de cada área. Todo esto, contribuye a generar procesos eficientes dentro de una organización.

A nivel nacional y regional, la diversidad de empresas que se encuentran, es amplia, tomando en cuenta el área o campo en el que se desempeñan y de igual forma el mercado y la necesidad de inversión en el país. Dentro de esta temática, una de las empresas que ha hecho los esfuerzos en cumplir con altos niveles de eficiencia y transparencia en su gestión de almacenes con éxito relativo, es Molinari \& Cacciaguerra Sucrs., C.A. localizada en la ciudad de Valencia, estado Carabobo, que surge como solución comercial para desarrollar actividades relacionadas con la compra, venta, distribución y comercialización de artículos de papelería.

Sin embargo, a pesar de tener como visión consolidarse en el mercado regional dentro del segmento en el cual presta sus servicios, mediante la optimización y mejora continua a través de procesos estandarizados que apunten a la satisfacción de sus clientes, la empresa evidencia debilidades en el manejo de sus almacenes. La política de calidad de la empresa se fundamenta principalmente en elementos como el mejoramiento continuo, innovación, economía y eficiencia, así como la calidad de los productos o servicios, a través de un profundo compromiso de todos y cada uno de los integrantes de la organización, así como una fuerte orientación a sus tareas, que de una u otra forma se va a derivar en una mayor productividad.

A pesar de lo antes descrito, una observación inicial de la situación de la gestión de inventarios de la empresa permitió determinar que tiene constantes problemas con las condiciones de almacenaje de producto, que genera devoluciones por daños en el estado de los productos, lo que se traduce en el incumplimiento de los compromisos adquiridos con sus clientes y la poca satisfacción de los clientes e incide en sus niveles de servicio.

Además, existe necesidad de mayor control de las existencias, ya que se presentan inconsistencias en la información registrada en los sistemas de la empresa (inventarios teóricos) y los inventarios reales. Asimismo, el Jefe de Inventarios de la empresa manifestó el interés de la organización por disminuir en forma continua los niveles de inventario, para mejorar la capacidad de almacenamiento de la empresa, con miras a alcanzar rotación adecuada de los productos, disminuyendo considerablemente la 
presencia de averías por daños en los productos y mal almacenaje, así como de merma, contribuyendo al mejor uso de los recursos humanos, materiales y económicos de la organización.

Del mismo modo, las inconsistencias en los inventarios que afectan la disponibilidad y fiabilidad del teórico en los productos de mayor demanda, por lo que es necesario que el trabajo de adquisiciones y las relaciones con los proveedores contribuya al flujo óptimo de la mercancías, en cuanto a su rotación; existen fallas en el manejo de la información, que equivale a mejorar el conocimiento de las tendencias de compras de distribuidores y clientes directos, por lo que también hay interés en implementar mecanismos de medición de la eficiencia de las operaciones a través de la implementación y evaluación de indicadores de gestión que permitan medir el desempeño de las actividades llevadas a cabo en la gestión de inventarios de la empresa.

De no llevarse a cabo una evaluación de la eficiencia de los procesos internos asociados a la gestión de almacenamiento, la empresa correría el riesgo de enfrentar el mercado sin las prácticas gerenciales adecuadas, atendiendo al cumplimiento de prácticas convencionales que no respondan a un plan de trabajo articulado, adaptado a la situación en que se desenvuelven los procesos operativos vinculados con el inventario y abastecimiento de empresas comercializadoras en Venezuela.

El presente estudio tiene como objetivo principal proponer estrategias logísticas para mejorar la gestión del almacén de la empresa Molinari \& Cacciaguerra Sucrs, C. A a partir de la situación inicial encontrada en la entidad, la cual ha presentado problemas logísticos a nivel de control de sus existencias, automatización de procesos, fallas en la planeación e información, herramientas de medición, así como desarrollo y formación del personal.

Para orientar a la investigación es importante plantease en primer lugar cuál es la situación actual de la empresa objeto de estudio en cuanto a la gestión de almacenamiento, cuáles elementos intervienen en la logística de almacenes de la empresa que puedan ser adaptados a una cadena de suministros eficiente y también cuáles acciones se pueden desarrollar para alcanzar la gestión eficiente en la logística de almacenes de la empresa Molinari \& Cacciaguerra Sucrs, C.A.; todo esto en virtud de determinar los factores claves que intervienen en la logística de almacenes de la empresa para desarrollar una gestión de almacenamiento y distribución eficiente, para finalmente proponer estrategias logísticas para el mejoramiento del almacén del producto terminado de la empresa Molinari \& Cacciaguerra Sucrs, C. A. que contribuyan con el logro de los objetivos de la entidad y su misión comercial.

Como bases teóricas que sustenten este estudio se asentarán los siguientes elementos:

\section{Descripción e importancia de la gestión estratégica}

El proceso de gestión estratégica de acuerdo a Hitt., Ireland y Hoskisson (2008:71), comprende "el conjunto tanto de decisiones, acciones y compromisos que requiere una empresa para lograr la competitividad estratégica deseada, basándose en estrategias integradas, diseñadas para utilizar las competencias centrales en ventajas competitivas", donde los líderes emplean el proceso administrativo estratégico a fin de lograr que las organizaciones lleven a cabo su visión y misión previamente definidas. 
De acuerdo con Robbins y Coulter (2005:104), "la gestión estratégica ofrece una dirección a la gerencia, reduce el impacto de los cambios, minimiza el desperdicio de los recursos y establece controles", por tanto, se hace necesario definir qué es la estrategia, la cual consiste en la determinación de la misión y de los objetivos básicos a largo plazo de una empresa, la adopción de cursos de acción y la asignación de los recursos necesarios para lograr estos fines. Todo esto implica selección de misiones, objetivos y de las acciones para cumplirlas. De este modo, los planes constituyen un método racional para el cumplimiento de los objetivos.

\section{Importancia de la Cadena de Suministro en las Organizaciones.}

Para lograr las metas organizacionales y cumplir con las expectativas de los consumidores o usuarios, las empresas deben cumplir con una serie de procesos, intercambio de materiales e información con diferentes entidades localizadas en diversos lugares, con la finalidad de ofrecerle al consumidor final, no sólo el servicio o el producto terminado, sino la satisfacción que éstos desean obtener. Dicho de otra manera, se puede considerar que las actividades logísticas son el componente operativo de la gerencia de la cadena de suministro; este componente incluye la cuantificación, la adquisición, la administración de los inventarios, el transporte y la gestión del flete, así como la recolección de datos y el reporte de la información. Steimberg (2009) hace la siguiente reflexión:

Los altos niveles de competencia en los mercados internacionales, han llevado a las empresas a la conclusión que para sobrevivir y tener éxito en entornos más agresivos, ya no basta mejorar sus operaciones ni integrar sus funciones internas, sino que se hace necesario ir más allá de las fronteras de la empresa e iniciar relaciones de intercambio de información, materiales y recursos con los proveedores y clientes en una forma mucho más integrada, utilizando enfoques innovadores que beneficien conjuntamente a todos los actores de la cadena de suministro (p. 45).

De esta forma, la gerencia de la Cadena de Suministro (también conocida como cadena de abastecimiento o cadena de valor, y mejor conocida en inglés como Supply Chain), se ocupa de asegurar que este proceso logístico funcione de la mejor forma posible. Jasso (2016:8) explica que "La cadena de suministro incluye todas las actividades de gerencia logística y por ello está presente en cada fase del proceso". Asimismo, el autor refiere que permite la gestión efectiva $y$, a través de los flujos de información, incrementa la calidad de servicio al cliente y de la cadena de valor, y que uno de los objetivos estratégicos en la administración de la cadena de suministros consiste en aumentar la capacidad de los participantes para tomar decisiones y formular planes.

\section{Planeación estratégica aplicada al componente logístico.}

Todas las empresas en mayor, o menor grado, cumplen procesos logísticos. La diferencia entre las compañías exitosas y las no exitosas, es que las primeras saben que la están haciendo y la toman como una herramienta indispensable para hacerse más 
competitivas. En un sentido amplio, se entiende por logística al conjunto de conocimientos, acciones y medios destinados a prever y proveer los recursos necesarios que posibiliten realizar una actividad principal en tiempo, forma y al costo más oportuno, en un marco de productividad y calidad, lo cual está en estrecha relación con el concepto de cadena de suministros. En este sentido, Jasso (2016), expresa:

Los procesos logísticos en la actualidad se han convertido en determinantes de éxito para las empresas, la logística coordina la utilización de recursos y optimiza los procesos dentro de una organización, buscando siempre la reducción de costos; el buen manejo logístico puede llegar a convertirse en una fuerte herramienta, por esta razón es importante para las empresas de cualquier sector tener claridad sobre sus procesos logísticos y el manejo de estos (p. 9).

En un sentido más concentrado, en el ámbito empresarial se tiene que logística, según Sallenave (2015:41) es "el proceso de gerenciar estratégicamente el movimiento y almacenamiento de materias primas, partes y productos terminados, desde los proveedores a través de la empresa hasta el usuario final". La logística, desde el punto de vista gerencial, es una estrategia necesaria para manejar de forma integral la cadena de suministros de una organización, de tal forma que logre el balance óptimo entre las necesidades del cliente y los recursos disponibles de la empresa y su desempeño debe ser medido a través del servicio al cliente final.

\section{Gestión de Almacén y Distribución}

El almacenaje y la distribución de productos ha sido objeto de estudios teóricos desde principios del siglo $X X$, dando como resultado una variedad de términos $y$ definiciones, relacionados con todo lo referente al flujo de materiales, entre estos están: logística, distribución, cadena de suministros, gestión de suministros y aprovisionamiento, entre otros. En 1963 surge en los Estados Unidos, el National Council of Physical Distribution Management (NCPDM o Consejo Nacional para la Gerencia de la Distribución Física), quien en 1976 emite el siguiente concepto para la distribución, citado por Ballou (2011):

\begin{abstract}
Gestión de la distribución física es una expresión que describe la integración de dos o más actividades con el fin de planificar, llevar a cabo y controlar, de una forma eficiente, el flujo de materias primas, inventarios en curso y productos terminados desde el punto de origen hasta el punto de consumo. Estas actividades pueden incluir, entre otras, el servicio al cliente, la previsión de la demanda, las comunicaciones de distribución, la gestión de stocks, la manipulación de materiales y de piezas de repuesto, la localización de plantas y almacenes, el aprovisionamiento de materiales y servicios, el empaquetado, la gestión de devoluciones, la evacuación de restos y desperdicios, el tráfico y transporte, y el almacenaje.
\end{abstract}

Cabe destacar que, en la actualidad, una de las partes más importantes en la distribución de productos terminados es el servicio logístico al cliente o la atención al cliente; a lo cual se refiere Anaya (2012) como 
"la cadena de actividades en torno a las ventas y que comienza con la recepción del pedido y finaliza con la entrega del producto al cliente, prolongándose en algunos casos como en el servicio de equipos, mantenimiento y cualquier otro soporte técnico".

\section{Eficacia y eficiencia en la gestión de operaciones de almacén y distribución}

Eficacia y eficiencia constituyen elementos básicos para cumplir con los objetivos propuestos en las organizaciones y su adecuada dosificación es condición fundamental para un liderazgo exitoso en la administración de almacenes. Por una parte, la eficacia, según Muller (2011) "mide los resultados alcanzados en función de los objetivos que se han propuesto, presuponiendo que esos objetivos se mantienen alineados con la visión que se ha definido", es decir, se logra mayor eficacia en la medida que las distintas etapas necesarias para arribar a esos objetivos, se cumplen de manera organizada y ordenada sobre la base de su prioridad e importancia.

El autor citado indica además que los cuatro criterios genéricos de la eficacia de la organización según un enfoque multidimensional son: el cumplimiento del objetivo (lograr los objetivos establecidos); la adquisición de recursos (reunir los insumos productivos necesarios); los procesos internos (crear y mantener sistemas saludables en la organización) y la satisfacción de los grupos estratégicos (lograr un mínimo de satisfacción en los tenedores de intereses más importantes). Estos aspectos llevados a la práctica fomentarán el logro de los objetivos propuestos mediante un plan estratégico, círculos de calidad, sobre todo en la necesidad de satisfacer al cliente.
Por otra parte, mientras que la eficiencia consiste, según Muller (2011) en "la medición de los esfuerzos que se requieren para alcanzar los objetivos". Entre otros, el costo, el tiempo, el uso adecuado de factores materiales y humanos, cumplir con la calidad propuesta, constituyen elementos inherentes a la eficiencia. Los resultados más eficientes se alcanzan cuando se hace uso adecuado de estos factores, en el momento oportuno, al menor costo posible y cumpliendo con las normas de calidad requeridas.

\section{Enfoques estratégicos en la planeación de las organizaciones}

El marco de oportunidades operativas que se pueden emplear para medir el desempeño en el manejo de las organizaciones, refleja el nuevo patrón bajo el cual se están desarrollando tales entidades, el cual puede resumirse de la siguiente forma, de acuerdo al análisis realizado por Malgiolio y Carazay (2012):

a) Existen funciones cruzadas en las empresas: Como consecuencia de la influencia del intercambio de información por medio de la tecnología, la organización funciona en la actualidad como un conjunto de procesos integrados, combinando los beneficios de la especialización con la velocidad, eficiencia y calidad de las nuevas relaciones entre los procesos.

b) Innovación: un efecto directo de la globalización de los mercados y el incremento en el nivel de conocimiento de los consumidores, es que los ciclos de vida de los productos se acortan, por ello, las empresas deben anticiparse a las necesidades futuras de los clientes, mediante la creación 
de nuevos productos y servicios, así como la implementación de mejoras continuas de los procesos.

c) Surgimiento de nuevas relaciones entre clientes y proveedores a través de la articulación de la cadena de suministro: la incorporación de tecnología de la información a los procesos organizacionales permite que las organizaciones en el presente integren los procesos de adquisición de materiales y producción.

d) Empleados de alto nivel intelectual: la incorporación de métodos de automatización y el incremento en la productividad en las últimas cuatro décadas, redujeron los niveles de fuerza laboral de trabajo directo, que realizaban funciones en los procesos tradicionales de la era industrial y que utilizaban mayormente sus capacidades físicas en detrimento de sus capacidades intelectuales.

\section{MATERIALES Y MÉTODO}

El presente estudio tiene características de investigación de campo, sustentada en un estudio documental, tomando la información directamente del lugar donde ocurren el fenómeno, en este caso, la situación actual respecto a las partidas en los pagos de las contribuciones en los clientes de la empresa sujeto de estudio.

Así mismo, la presente investigación es descriptiva, ya que obtienen resultados mediante descripción de determinadas situaciones asociadas al fenómeno particular (estrategias logísticas al inventario de mercancía) dentro del grupo social específico (Integrantes del área de inventario y almacén de la empresa Molinari \& Cacciaguerra Sucrs. C. A.).

Las unidades de análisis están constituidas por el personal que participa en la gestión de almacén e inventario de la empresa, en un total de seis personas que se incluye en su totalidad dentro de la muestra. Las técnicas de recolección de información son la observación directa, a través de la lista de control o guía de observación, también denominada lista de cotejo y la encuesta. En el caso de la técnica de encuesta, el instrumento seleccionado es el cuestionario.

\section{RESULTADOS Y DISCUSIÓN}

Existe la noción generalizada por parte de los socios o accionistas de pequeñas y medianas empresas, que el proceso de aplicación de estrategias para mejorar sus procesos internos, es una actividad que tiene relación con el manejo y los recursos de grandes empresas. Sin embargo, la importancia que reviste esta área para la gestión de las organizaciones, hace que sea muy necesaria la pronta familiarización de este concepto por parte de estos empresarios, ya que la implementación de mejoras mediante estrategias contribuye con los logros del área de inventario de la empresa a mediano y largo plazo, y a su vez, permita adaptar a la organización a los cambios que ocurren en el entorno socioeconómico y comercial del país, contribuye a alinear al equipo humano, y define los compromisos claros sobre lo que va a hacer la empresa en el futuro, en coherencia con los objetivos fundamentales de la empresa.

La realización del proceso de observación permitió evidenciar la ausencia de los siguientes elementos, que inciden negativamente en la gestión de almacenamiento de la organización y 
que deben ser consideradas dentro del plan de mejoras basado en estrategias logísticas en la gestión de almacenamiento. En resumen, se determinó que a pesar que existen estrategias y objetivos básicos de la planeación de distribución en la entrega de productos terminados a los clientes, no se cumple el resto de actividades, lo cual se nota en hechos como que se carece de alineación de objetivos y estrategias, no existe comunicación de la estrategia hacia los trabajadores; tampoco existen objetivos medibles consecuentes con las estrategias ni se hace una revisión periódica de las metas.

En líneas generales, es importante la creación de estrategias logísticas con la finalidad de incrementar la eficiencia de los resultados y la información generada por la gestión de almacenamiento de la empresa estudiada, para que ésta pueda ser transmitida con los mayores niveles de confiabilidad y garantizar la disponibilidad y entregas al área de producción. Es importante, en este sentido, asignar la responsabilidad de controlar y verificar los procesos de almacenamiento para medir su eficiencia y tomar las medidas pertinentes que orienten su mejoramiento, así como coordinar la consecución más idónea de los recursos tecnológicos, financieros, materiales y humanos involucrados en la actividad relacionada con la gestión de almacén.

En relación a la logística de inventarios, se tiene la percepción que el desempeño actual del departamento de almacén no contribuye con el crecimiento económico de la organización; no se toman en cuenta los principios de eficacia y eficiencia para llevar a cabo las actividades en el departamento de almacén de la empresa. Los resultados infieren que en la actualidad no se le ha dado mayor importancia a la mejora continua dentro de la gestión de almacenamiento de la organización, ya que al identificar aquellas incidencias que puedan producir una pérdida de fiabilidad en la información, contribuye a satisfacer la rendición de cuentas, prestando especial atención a los requerimientos legales que puedan afectar el régimen económicofinanciero de la empresa.

En referencia a las estrategias logísticas, la mayoría de las personas incluidas en la muestra tienen la percepción de que en la empresa no existen objetivos definidos de la gestión de Almacén de la empresa que permitan servir como referencia al trabajador en el logro de metas individuales y colectivas; asimismo, la forma como el supervisor inmediato organiza y dirige la gestión de almacenamiento de la empresa no ayuda a facilitar el trabajo, lo que permite concluir que la implementación de una estructura orgánica funcional para la empresa debe estar apoyada por la participación de todo el personal, de forma tal que los líderes de áreas y sus respectivos equipos cumplan con las responsabilidades asignadas, entre las cuales cobra especial importancia el control y la supervisión de las actividades.

Además, en la empresa unidad de estudio; en la actualidad no se desarrollan las actividades del Departamento de Almacén en un ambiente que promueva la mejora continua de los procesos; en la actualidad no existen en el departamento de Almacén estrategias orientadas a satisfacer las necesidades de información de la empresa, además que los recursos tecnológicos empleados por el Departamento de Almacén no responden a las necesidades y exigencias de crecimiento de la empresa. 


\section{CONCLUSIONES}

El análisis de los datos obtenidos indica que el personal consultado tiene la percepción de que la empresa carece de estrategias de mejoramiento continuo orientadas a la optimización de la gestión de almacenamiento. Estos resultados traen consecuencias negativas para la organización, ya que al no aplicar la mejora continua se limita la capacidad de innovar, de anticiparse a los requerimientos del mercado y la posibilidad de mejorar los resultados, pues si se siguen aplicando los mismos métodos es improbable obtener nuevos resultados, por lo que la mejora continua de la capacidad y resultados, debe ser el objetivo permanente de la organización.

Tomando en cuenta estos elementos, uno de los métodos que reviste mayor importancia en la actualidad, es la aplicación de estrategias orientadas a mejorar el control interno de los procesos de almacén e inventario, como un instrumento práctico para que esta organización efectúe su gestión en la disposición de los recursos y a su vez, evaluar alternativas de mejoras en los procesos, y haga cumplir los lineamientos establecidos por la dirección en los niveles tácticos y operacionales.

En líneas generales, se propone establecer un instrumento gerencial que permita hacer más eficiente el proceso de registro, control y toma física de los inventarios de productos terminados y materias primas, así como la distribución adecuada de tareas y responsabilidades entre las áreas que participan en este proceso; además de mantener el registro y documentación oportuna y suficiente de los movimientos que se realicen durante los procesos.

Por lo que se propone a la empresa, objeto de este estudio:
- Evaluar y aplicar los procedimientos que se detallaron en todo el ciclo de abastecimiento, a fin de garantizar un eficiente desarrollo de este proceso.

- Implementar la estrategia propuesta, la cual está diseñada y adaptada a los procesos de la empresa; para ello se sugiere informar a las áreas involucradas. Esta estrategia deberá ser revisada periódicamente para realizar las modificaciones que se consideren pertinentes, a fin de mantener su vigencia en el tiempo.

- Desarrollar e implementar una estrategia de gestión de almacén, que permita a la empresa contar con una herramienta que documente los procesos y los pasos a seguir en el ciclo de recepción y despacho de la mercancía. Asimismo, segregar las funciones para la adquisición, control, almacenaje y distribución de los inventarios de la empresa.

- Se recomienda, contar con un grupo de trabajo que se encargue exclusivamente del almacén de materias primas, con el fin de que exista una adecuada segregación de funciones, y la responsabilidad de la custodia y control de inventario forme parte de su rol como trabajadores. Esto evitará posibles errores en la recepción y despacho de la mercancía e irregularidades que se pudieran presentar, como, por ejemplo: hurto o pérdida intencional del inventario.

- Realizar talleres de capacitación, motivación, mejorar el ambiente de trabajo y comunicación entre las áreas relacionadas con la finalidad de reforzar los conocimientos e incentivar al personal 
reconociendo los logros por metas establecidas.

- En el área de inventarios, establecer controles internos específicos y evaluarlos periódicamente para que su cumplimiento sea eficiente, asimismo se debe capacitar constantemente al personal, en especial al de reciente ingreso, para evitar pérdidas causadas por desconocimiento de las normas y procedimientos establecidos.

- A futuro, divulgar y fomentar la práctica de la auditoría operacional con enfoque de auditoría interna de la gestión de almacén, para que la empresa, obtengan tanto beneficios como ahorros financieros, fortalecimiento del control interno, corrección oportuna de procedimientos operacionales que puedan dañar y limitar el desarrollo económico de la empresa.

\section{REFERENCIAS}

Anaya, Juan José (2012). Logística integral. La Gestión Operativa de la Empresa. España: Editorial ESIC

Ballou, Robert (2011). La Logística Empresarial. (1era edición). Madrid, España: Editorial Díaz De Santos

Bohórquez, Ella y Puello, Roy (2013). Diseño de un modelo de gestión logística para mejorar la eficiencia organizacional de la empresa Coralina \& Pisos, C.A., Trabajo de Grado. Cartagena: Universidad de Cartagena, Colombia

Cano, M. y Olivera, D. (2009). Algunos modelos de planeación estratégica. Publicaciones de Ciencias Administrativas del Instituto de Investigaciones y Estudios Superiores de las Ciencias Administrativas de la Universidad Veracruzana, México

Chase, Richard; Jacobs, Robert y Aquilano, Nicholas (2009). Compras Importadas de operaciones: producción y cadena de suministros. Duodécima edición. McGraw Hill Editores, Ciudad de México

Donnelly, Iván. (2007) Fundamentos de Dirección y Administración de Empresas. Octava Edición. Editorial Trillas, Ciudad de México

Hernández, R. Fernández, C. y Baptista, P. (2015) Metodología de la Investigación. McGraw Hill Interamericana de México, Naucalpan de Juárez, Edo. de México

Hitt Michael, Ireland, Duane y Hoskisson, Robert (2008). Administración Estratégica. Competitividad y Globalización. Madrid: Séptima Edición. International Thomson Editores, S.A

Jasso, Javier (2016). La competitividad internacional empresarial: intensidad patentadora y de mercado. Ciudad de México: McGraw Hill Interamericana

Koontz, W. y Weihrich, H. (2014). Administración: una perspectiva global. $12^{\mathrm{a}}$ edición. México: McGraw-Hill

Malgioglio, J. y Carazay, C. (2012). Distintos Enfoques del Capital Intelectual. Rosario: Publicaciones de las Séptimas Jornadas "Investigaciones en la Facultad" de la Universidad Nacional de Rosario, Argentina Mendoza, J., Hernández, M. y Tabernero, C (2011). Retos y oportunidades de la investigación en marketing interno. Revista de Ciencias Sociales (Ve), vol. XVII, núm. 1, enero-marzo, 2011, pp. 110-125. Maracaibo: Universidad del Zulia

Miranda, Roberto (2016). Sistema General de Gestión del Conocimiento Estratégico. Revista Espacios, Vol. 29 (2), Caracas

Mintzberg, Henry (2008). Reflexiones sobre Planificación Estratégica. Ciudad de México: Editorial Prentice Hall

Mora, Luis (2008). Indicadores de la Gestión Logística. Ecoe Ediciones. Bogotá, Colombia

Muller, M. (2011). Fundamentos de la administración de inventarios. Bogotá, Colombia. 
Nonaka, I. y Takeuchi, H. (1999). La organización creadora de conocimientos. Editorial Oxford, Ciudad de México

Pau, J. y Navascués, R. (2013). Manual de Logística Integral. Editorial Díaz de Santos, España

Reyes, J. (2015). Propuesta de lineamientos estratégicos para optimizar el proceso de almacenaje de la empresa Conduven, C.A. Ubicada en la Victoria

Rodríguez, G. (2009) Comunicación Interpersonal y Comunicación Organizacional. Centro de Información. Conindustria- Programa Coninpyme. Caracas
Robbins, Stephen y Coulter, Mary (2005). Administración. Ciudad de México: Editorial Prentice Hall

Sallenave, Jean Paul (2015). La Gerencia Integral ¡No le tema a la Competencia, Témale a la Incompetencia! Caracas: Editorial Norma

Serna Gómez, Humberto (2009). Gerencia Estratégica. Global Ediciones. Bogotá

Steimberg, Fred (2009) La nueva teoría del comercio internacional y la política comercial estratégica. McGraw Hill Editores, Ciudad de México 